\title{
Keyword Index for Volume IIO
}

${ }^{18}$ F-FAMT 1985

${ }^{18}$ FLT-PET 875

25-hydroxyvitamin D 2772

3,5-diethyl-1,3,5-thiadiazinane-2-thione (DETT) 63

3DPCR 2615

3-hydroxy-3-methylglutaryl coenzyme 802

4-hydroxy tamoxifen 1488

5-aza-2'-deoxycytidine 1923

5-fluorouracil 946

$5 \mathrm{hmC} 668$

$8 \mathrm{q} 24164$

\section{ABCB1 1673}

acceleration 2217

accreditation 850

actinic keratosis 520

active surveillance 2405

activin signalling 2604

acute myeloid leukaemia 783

ADAM9 2945

ADAMs 1535

adaptive design 1950

ADCC 1221

adenocarcinoma 1571, 2688

adherence 831

adhesion 146, 753

adjuvant chemotherapy 313

adjuvant therapy 1133,1545

adolescent 1342

advanced adenoma 1228

advanced biliary tract cancer 2165

advanced neoplasia 1334

aerobic exercise 831

AFP 2090

AFP-L3 2090

age 1125

aggressiveness 1767

Akt 399, 935, 946, 1785, 1288, 2081

Akt signalling 899

albumin 2090, 2277

alcohol 242

aldehyde dehydrogenase 3A1 2593

ALDH1 430

ALDH1A1 protein 2935

aldo-keto reductase 1C3 1506

alkylating agents 2623

allergy 1825

alpha9 1269

alpha-fetoprotein 2277

amino-acid transporter 2030

amoeboid phenotype 2099

amphiregulin 648

amplification 2914

androgen receptor gene amplification 1655

aneuploidy 2159

angiogenesis $26,107,115,469,741,946$,

$1027,1034,1179,1269,2700$

angiogenic cytokines 441

animal welfare 928

anoikis 958

anti-angiogenesis 602,882

anticipated delay 12

anti-inflammatory agents 1409

anti-oncomir 189

antitumour activity 1733

anti-tumour immunity 83

anxiety 868

APOBEC3 2615 apoptosis $63,164,341,421,656,753$, $1645,2144,2224$

apoptosis and cytotoxicity 146

appetite loss 1409

aromatase inhibitors 1133

ascites fluid 2269

ASCT2 2030, 2506

Asian 2187

aspirin 2378

atopy 1825

autoimmune disease 1825

autophagy 899, 2209

awareness 12

axitinib 875, 2821

B7-H3 2072

BAP1 1058

Barrett's oesophagus 775, 2378

basal cell carcinoma 1855

BCAC 535

BCAS2 2144

$\beta$-catenin 935,2708

BCH 1985

Bcl-2 946, 1785

benchmarks 1917

benign behaviour 286

benign breast lesions 788

benzene 783

betaine 808

big five 1820

bilateral 1053

biliary cancer 882

bilirubin 2090

biliverdin 2116

biodistribution/toxicology 2441

biology 1985

biomarker strategy design 1950

biomarkers $26,107,123,565,648,679$, 928, 976, 1139, 1236, 2081, 2090, 2109, 2178, 2201, 2277, 2283, 2291, 2462, 2524, 2677, 2700, 2765, 2935 BIRC5 protein 2935

bladder cancer $679,842,1767,1862$, $1871,2123,2945$

blue dye 2837

BMP-6 1634

BOADICEA 535

body mass index 1133

bone marrow stroma 2099

bone metastasis 1634

borderline behaviour 286

borderline/mildly dyskaryotic cytology 1579

bortezomib 656

bowel cancer 1705

bowel cancer screening 1705

BRAF 1045, 1359

$B R A F$ mutation 1228

brain cancer 242, 1825

brain tumour 2354

BRCA1 530, 535, 1081

BRCA1/2 1074

BRCA2 530, 535, 1081

BRD4 1189

breast cancer $4,146,230,535,560,565$, $573,715,724,831,859,916,1081$, $1088,1133,1288,1367,1378,1420$, $1681,1688,1744,1898,1908,1958$, 2072, 2144, 2195, 2209, 2327, 2412,
2524, 2623, 2765, 2772, 2847, 2855, 2905

breast cancer cells 1298, 2514

breast cancer incidence 224

breast cancer metastasis suppressor gene-1 2054

breast cancer risk 224, 2339

breast cancer stem cells 2063

breast conserving surgery 1420

breast density 1908

breast screening 556

breastfeeding 1074, 1367

BRMS1 2054

bromodomain 1189

bronchitis 2796

CA-125 kinetics 1517

cachexia 1409

CAFs 724

calretinin 2040

cAMP 1278

cancer $479,584,1820,1936,2321$

cancer epidemiology 7

cancer genomics 2615

cancer hallmarks 2935

cancer metabolism 330

cancer prevention 2361

cancer research 928

cancer risk 2361

cancer stem cell 430

cancer survival 1359

cancer survivors 868

cancer testes antigens 2450

cancer therapy 2496

cancer-associated fibroblast (CAF) 469

cancer-specific antigens 2450

cancer-stroma interaction (CSI) 469

capecitabine 1438

carbohydrate 1316

carbon ion radiotherapy 2389

carboplatin 256, 1923, 2975

carcinoma in situ 260

carcinoma of unknown primary 208

cardiovascular 1338

carotene 792

case-control study 808, 1385, 2348

caspase 656

castration-resistant prostate cancer 2201, 2472

Caucasian 55

CCL7 (MCP-3) 686

CCNC 2246

CCR2 686

ccRCC 1778, 2261

CD105 1778

CD26 2232

CD43 146

CD44 916, 958, 1793

CD74 2040

CD8 2551

Cdc20 2905

CDH1 1053

CDK1 510

CDK19 2246

CDK8 2246

CDK8 submodule 2246

CDK9 1189

CDKN2A 2985

CEACAM1 1244 cell cycle 656, 2434

cell cycle-specific transgene expression 94

cell growth 2583

cell migration 706, 2569

cell-free DNA (cfDNA) 363, 2054

CellSearch system 375

cellulitis 2796

central venous access catheters 1438

cervical cancer $34,278,1260,1748$

cervical cytology 1841

cervical intraepithelial neoplasia 1579

cetuximab 648

cetuximab resistance 2887

c-FOS 753

chemokine 741, 1179, 1992

chemokine receptor 1992

chemoprevention 2378

chemoresistance 1785, 1958, 2881

chemosensitivity 479

chemotherapeutic sensitivity 2269

chemotherapy $49,271,479,908,1488$, $1759,2165,2462$

chemotherapy resistance 2489

child 1342

childhood 1338

childhood leukaemia 1402

Chloroquine 899

cholangiocarcinoma 882

cholecystectomy 2348

choline 808

choline kinase-alpha 330

ChREBP 715

chronic lymphoid leukaemia 783

CIMBA 535

CIN 2159

circulating endothelial cells 1236

circulating microRNAs 2462

circulating tumour cells 375

CIS 668

cisplatin $341,625,1163,1733,1785$

cisplatin resistance 123

CK19 2544

classification 1688

clear cell adenocarcinoma 2881

clear cell RCC 2537

clinical 1688

clinical features 2633

clinical outcome 459

clinical trials 551

cluster randomised controlled trial 2829

c-Met 1307

c-MYC copy number gain 2688

CNS tumour 286

coculture 1552

cohort 584, 2321

cohort study 214, 249, 1316, 2339, 2354

collagen I 409

colon 459

colon cancer $435,459,469,817,1211$, 2081, 2310, 2544

colon neoplasms 2935

colorectal 648

colorectal adenoma 249

colorectal cancer $156,164,450,530,831$, $875,935,946,1001,1008,1014,1221$, $1316,1359,1595,1606,1614,1847$. 2159, 2178, 2300, 2551, 2716, 2728

colorectal cancer screening 1334, 1705 colorectal neoplasms 850 
communication 1101

co-morbidity 573

competing risk 1378

computed tomography 2354

concordance 1950

conditionally replicating adenoviruses 2496

consultation aid 1101

contralateral prophylactic mastectomy 1081

core biopsy 2195

Coronin 1A 1622

cost-effectiveness 1841, 2829

covariates 304

C-reactive protein 183

cross-talk 1199

CRPC 1634

cutaneous squamous cell carcinoma 520

CXCL12 1179

CXCL16 1535

CXCR4 686, 1179, 1288, 2261

CXCR6 1535

CXCR7 1179

cyclic adenosine mono-phosphate 1278

cyclooxygenase inhibitors 2378

cyclophosphamide 1733

cystectomy 1759

cysteine 71

cytidine deaminase 2615

cytochrome P450 3A (CYP3A4) 2669

cytokine receptor 133

Cytokine-induced Killer (CIK) cell 1992

cytokines 133, 733

cytostatic drug 625

DAP3 421

database 584

DCP 2090

dedifferentiation 2923

deep venous thrombosis 1118

depression 868, 1409

depressive symptoms 593

derived neutrophil-lymphocyte ratio 2531

dFdCTP 304

diabetes $1825,1847,2361$

diagnosis 584,1748

diagnostic algorithm 1244

diagnostic interval 584

diagnostics 976

diet 1392

dietary intake 808

dietary pattern 2785

differentiation 199

differentiation status 520

diffusion-weighted imaging (DWI) 107 diffusion-weighted MRI 616

dihydropyrimidine dehydrogenase 2728

discordance 2195

discrete choice experiment 859

disease control 172

disease progression 2537

disease subtypes 1088

distant metastasis 1378

DJ-1 (PARK7) 1298

DLBCL 369

DNA damage response 1014

DNA demethylation 668

DNA methylation 775, 1923, 2054, 2123

DNA repair gene mutations 1663

DNMT3B 2131

docetaxel 894, 2170, 2462, 2472, 2669

Dock7 1307

dose intensification 1413

dosing schedules 1977

double and triple mutations 2812 drug resistance 1923, 2896

drug sensitivity 330

drug therapy 1943

drug-drug interaction 888

dual-imaging of virus and host cells 94

DVT 1118

dynamic contrast-enhanced magnetic

resonance imaging (DCE-MRI) 107

E2 1488

early breast cancer 2187,2450

early diagnosis 584, 1001

EBRT 2396

E-cadherin 1053

effectiveness 421, 551, 1841

efficacy 551, 1977

EGFR 648, 2116, 2688, 2716

EGFR amplification mutation 1045

EGFR and HER2 tyrosine kinase

inhibitor 2647

EGFR mutation 55

EIF5A2 399, 1767

elderly 313

elective nodal irradiation 1420

embryonic stem cells 2131

$\mathrm{E} \mu-m y c 928$

EMT 156, 695, 724, 1958, 2000

EMT/MET 1497

EMVI 19

endometrial biopsy 609

endometrial cancer 609, 2640, 2874

endometrial cancer staging 609

endometrial carcinoma 107

endometrial carcinoma grading 609

endometrial histology 609

endometrial sampling 609

endometriosis 1878

endoplasmic reticulum stress 1488

endothelial 1778

endothelin B receptor 1027

england 1322

EpCAM 679

EPCAM protein 2935

epidemiology 242, 797, 1367, 1392, 1891

epidermal growth factor receptor 882

epigenetic silencing 636

epigenetic-gene interaction 2123

epigenetics 2874

epiregulin 648, 2716

epithelial ovarian cancer 42

epithelial-mesenchymal transition

(EMT) 958, 1298, 1767, 2514, 2569

ERBB2 2985

EREG 648

eribulin mesilate 1497

ERK1/2 1034, 1288

$\operatorname{ERR} \beta 2144$

ESMO 2640

estimates of effect 1834

ethnic 1322

ethnicity 4, 224, 230, 1433

ex vivo model 2604

expanded access 1721

expression microarray profiling 520

external quality assessment 850

external validation 42

Ezrin 695

faecal immunochemical test 1334

FAK 2747

familial prostate cancer 1663

family 1074

family history of breast cancer 2339

Fascin-1 2300

fatigue 868,1409
FDG PET/CT 2209

female breast cancer 1891

FENSAM 2109

fetal germ cells 668

FGF receptors 1088

FGFR amplification 967

FGFR1 2914

fibrinogen 984

fibroblast growth factor receptor 320

fibrosarcoma 1456

FISH 1655

FIT 1334

flavopiridol 1189

FLT-PET 2847

fluid intake 797

fluorescence in situ hybridisation 1571, 2688, 2914

fluoropyrimidine 2728

folate 1328,2327

FOLFIRI 875

FOLFOX 875, 1438, 2662

follow-up 19,859, 2420

food frequency questionnaire 797

four-factor model 278

FOXO1 1260

FoxP3 1552, 2551

Foxp3+ regulatory $\mathrm{T}$ cells 459

free light chains 2277

FSCN1 189

FST 2144

fucosyltransferase 156

GA201 1221

Gabl 1307

galectin-1 1250

galectins 741

gallbladder cancer 882

gallstones 2348

gambogic acid 341

gastric cancer 271, 421, 967, 1169, 1199,

$1211,1328,1552,1801,2011,2021$,

2283, 2291, 2662, 2923

gastro-oesophageal adenocarcinoma 1525

GC 1244

G-CSF 133, 1211

G-CSFR 133, 1211

gefitinib 55

gemcitabine 83, 172, 304, 1481, 1943, 2472

gender 1385

gene expression 2662, 2728, 2738

gene expression profile 1244

gene expression profiling 1525

gene function 1552

genetic markers 2537

genetic risk 2623

genistein 1645

germ cell tumour 2738

germline mutation 1053

GIST 1155

glioblastoma 1179, 1307

glioma 242, 1825, 2560

global methylation level 2765

Glut-1 715

glyco-engineering 1221

glycosylation 2021

GNA11 1058

Golgi phosphoprotein-3 (GOLPH3) 2250

groin node 2837

growth 2300

growth factor receptors 1169

guaiac faecal occult blood test (gFOBt)

guidelines 831

haeme oxygenase-1 341

haemolysis free 1001

HAGE 2450

haptokinesis 409

haptotaxis 409

$\mathrm{HbA}_{1 \mathrm{c}} 2361$

$\mathrm{HBx} 1066$

HCC 1811

hCG $\beta 1759$

head and neck cancer 501, 593, 2677

head and neck tumours 1587

health status 573

health-related quality of life 593 , 2427

help-seeking 12

hepatectomy 1110

hepatocellular carcinoma 733, 1066, 1110, 2090, 2277

hepatocellular carcinoma subclasses 2708

HER2 384, 625, 2209

HER2 amplification 967

HER2-positive 2756

HER2-positive breast cancer 1139

HER3 384

HERBIS 1163

herpes zoster 2796

HGF 2716

HIF- $1 \alpha 115,1250,1066,2209$

high risk 1074

high-grade glioma 2655

histological grade 2195

histology 1385

histomorphological marker 107

histonedeacetylase inhibitor 656, 1155

histopathological regression 1712

HLA Class I expression 459

HLA-E 459

HLA-G 459

head and neck squamous cell carcinoma (HNSCC) 2116, 2887

Hodgkin lymphoma 868

hormonal therapy 1133

hormone naïve 1655

hospitalisation 1342

HPV 1561, 1579, 1587, 1793

HPVE7 1034

HSF1 71

Hsp 9071

human papillomavirus 489, 1587, 2369, 2804

Human teratocarcinoma stem cells 2131

humane end points 928

hyperglycaemia 2361

hypermethylation 2874

hypermutation 2615

hypertension 797

hypofractionation 2217

hypoxia 172, 715, 935, 1014, 2865

ICAM-1 1801

ID-1 1298

ifosfamide 888

IGFBP 2855

IGFBP7 775

IGFs 2865

IL15 1673

IL-6 2965

IL-8 2965 
immunohistochemistry 775,1525 , 1571

immunosuppression 2965

immunotherapy 1472

immunotherapy vaccine 2450

impact 2804

improvement 1936

incidence 4, 535, 797, 1322

incidental breast cancer 788

infection 2796

infectious diseases epidemiology 7

inflammation 208, 435, 1930, 2524

inflammatory conditions 1825

inflammatory parameter 369

information exchange 1101

infusion rate 304

inguinofemoral lymphadenectomy 2837

inhalation 1427

inhibitors 1506

Insulin-like growth factor 2855

integrin 1269

integrin $\alpha 5764$

intensity-modulated radiotherapy (IMRT) 49, 297

interaction 1359

Interlerukin-6 (IL-6) 469

interleukin-2 1427

interleukin-24 2496

interleukin-8 2047

intermediate-risk group 278

international metastatic renal cell cancer database consortium 1917

interphase-FISH 2537

interval cancer 556, 560

intervention 593

intratumoural budding (ITB) 1008

intratumoural subsites 255

invadopodia 189

invasion 399, 1298, 1307, 2011, 2945

invasive lobular breast cancer 1053

ipilimumab 1721

iRGD peptide 1481

irinotecan 1148, 1733

irradiation 1179

JAK/STAT 133

Japan 1316

JMJD2B 1014

KDR 2700

Keap1 1785

keratinocyte 199

Ki-67 1561, 2847

Ki67 promoter 2496

kidney cancer 1622

kinase and phosphatase inhibitors 2441

KIT 1155

KLF17 1298

KLF4 450

K-PD modelling 1517

K-RAS 648, 1606, 20472874

KRAS gene mutation 1148

lactate dehydrogenase inhibitors 172 laparoscopic radical cystectomy 842 lapatinib 1139, 2647

laryngitis 2796

LASSO 2109

LAT1 1985, 2506

late complications 297

late effects 1338

late toxicity 49

latent class modelling (LCM) 2756

LBH589 1155

LDH5 2217

LDHA 2217 leptin 1525, 1801

leukaemia 1506, 2354

Lgr5 2011

ligands 2716

likelihood functions 34

LINE-1 repetitive sequences 2123

liposarcoma 1456

liquid chip technology 2812

liquid chromatography tandem mass spectrometry 2489

lobular carcinoma in situ 1053

local 1456

local recurrence 297

localisation 1712

locally advanced breast cancers 1413

long noncoding RNA 164

long-term survivors 2756

long-term use 249

low-dose radiation exposures 214

LRIG1 1793

lung 1936

lung adenocarcinoma (AC) 991, 2047, 2583

lung cancer $341,764,1385,1834,2427$, 2688

lung carcinoid 1244

lung carcinoma 1545, 1571

lung neoplasm 908

LVSI 2640

lymph node 2544

lymph node and distant metastases 1008

lymph node excision 34

lymphatic metastasis 34

lymphocyte subsets 2551

lymphocytes 435

lymphoid malignancies 2796

lymphoma 928

lysate microarray 2072

magnetic field 1402

magnetic resonance elastography 1727

magnetic resonance imaging 1465

magnetic resonance spectroscopy 625

male 1744

male breast cancer 1891

malignant mesothelioma 510

malignant pleural mesothelioma 984 2040, 2232

malignant progression 715

mammary gland 2747

mammary tumour 2747

mammographic density 1898, 1908

mammography 560, 2187, 2412

mammospheres 2063

MAPK pathway 520

Markov model 2412

mastectomy 2187

mathematical model 7

mathematical modelling 1517

matrix metalloproteinase 916

MDM2 656

MDSCs 83

MED13 2246

Mediator 2246

medulloblastoma 636

melanoma $399,802,1427,1721,1855$

2489,2496

menarche 2339

Merkel cell carcinoma 1446

Merkel cell polyomavirus 1446

mesenchymal stem cells 1199

mesenchymal-epithelial transition 2514

mesothelioma 2479

meta-analysis $817,1595,1820,1862$,

$1878,2327,2369,2378,2772,2785$ metabolism 363

metastases 441

metastasis $256,392,450,686,695,741$,

$753,764,916,1288,1497,1748,2000$ 2011, 2072, 2099, 2144, 2300, 2310, 2537

metastasised 2738

metastatic 1125

metastatic breast cancer 375, 384, 2756

metastatic colorectal cancer 1148, 1438

metastatic prostate cancer 2462

metastatic renal cell carcinoma 1917

methylation 808

methylation-specific PCR 2054

mGPS 1930

MIB-1 2217, 2583

mice 928

microRNA 353, 991, 1199, 1614, 2072, 2945

microRNA-133b 2310

microsatellite instability 1228

microvascular density 2700

midline carcinoma 1189

MIF 2040

migration 146, 1801, 2865

minimal residual disease 2544

miR-126 2945

miR-1260b 1645

miR-145 2300

miR-196a 1260

miR-22 1250

miR-29a 450

miR-7 353

miR-9 392

MIRA 2224

miRNA 764, 1260, 2291

miRNA-9 636

mismatch repair 2728

MMP14 189

MMPs 441

MMSET 2662

model 2640

modelling 1834

molecular 1688

molecular subcategories 146

molecular subgroups 2551

monoclonal antibody 1221, 1472

morbidity 2837,2772

mortality reduction 2412

mouse model 894, 946

MRI 625

MSC 2955

MSI 1359, 2159

mTOR inhibitor 1917

multi-arm trials 2178

multi-colour FISH 2985

multidisciplinary 1936

multimodality therapy 984

multiphase contrast-enhanced MRI 616

multiple myeloma 63

multiplex testing 2812

multivariate analysis 1712

multivitamin 249

mutant p53 2569

mutation 1066

MYC 2985

myeloma 2224

NADPH oxidase 2569

$\mathrm{Na}^{+} / \mathrm{H}^{+}$exchanger 1916

nasopharyngeal carcinoma 49, 297, 392 808,2633

nasopharyngeal neoplasms 1465

NDC80 510

neoadjuvant 430, 1139

neoadjuvant chemoradiotherapy 19

neoadjuvant chemotherapy 421, 1413,

1420,2847

neoadjuvant treated 1712

neoplasms 1342

neoplastic progression 2378

network meta-analysis 2396

neuroendocrine tumours 115

neuropilin-1 1481

neutropenia 27

next-generation sequencing 1663, 2738

NF- $\kappa$ B 63, 341, 656, 695, 2965

NICE guidelines 584

nicotine 1785

NLR 1930

nomogram 34, 1110, 1943, 2261

non-coding RNA 1614

non-malignant 286

non-melanoma 802

non-small cell lung cancer (NSCLC) 55 ,

$353,363,602,991,1236,1930,2030$,

2054, 2812, 2914

non-uptake 1705

Notch1 2283

NRF2 71

NSC23925 2896

nuclear 199

nuclear workers 214

nullipotent 2131

Nur77 935

nurse 593

NUT 1189

OATP1B 894

observational research 551

oesophageal adenocarcinoma 775, 2378, 2985

oesophageal cancer 1027, 1328, 1985

oesophageal squamous cell carcinoma 189, 2109, 2369, 2785

oesophagogastric adenocarcinomas 1712

oestradiol 1898

oestrogen receptor 1488

older 573

ombrabulin 2170

omega 6 1392, 2099

omega-3 1392

oncology 1101

oncotherapy 83

one-carbon metabolism 2123

one-carbon metabolism pathway 1328

open radical cystectomy 842

oral administration 1269

oral anticoagulant 1334

oral bioavailability 2669

oral contraceptives 1074, 1367

organic food 2321

oropharyngeal cancer 489

oropharyngeal tumours 1587

orthotopic ileal neobladder 842

orthotopic mouse model 146, 2000

OS 181

OSNA 2544

osteopontin 1269

osteosarcoma 2896

outcomes 4, 551, 1456, 1688

outcomes assessment 1917

ovarian cancer $123,133,330,625,976$

$1074,1118,1392,1535,1878,1923$, 
3010

over-diagnosis 1841, 2412

oxaliplatin 1733, 2472

p16 1561, 1587, 1793

p16ink4a 1587

p16/Ki-67 dual-stained cytology 1579

p185HER2-positive metastatic breast cancer patients 1968

p2 $7^{\text {Kip1 }} 1260$

p53 656, 2224, 2747

p95HER2 overexpression 1968

PA28gamma 353

paclitaxel 271, 1488, 1958, 2647, 2669, 2896, 2975

paediatric ALL 1673

paediatric cancer 636

pain 1409

pancreatic adenocarcinoma 1698

pancreatic cancer $183,313,320,1481$, 2348

pancreatic cancer cells 409

pancreatic ductal adenocarcinoma 172, 2269

pancreatic neoplasm 1943

pancreatic stellate cells 409

panobinostat 1155

parity 1074

PARP inhibitor 1977

pathogenesis of testicular cancer 2604

patient choice 573

patient preferences 859

patient survival 399

patient-reported outcomes 2427

patients' outcome 1968

pazopanib $26,888,2647$

PC 1645

PDE4D 1278

PDE4D7 1278

PE 1118

peer 1936

peer review 850

pelvic lymph node 1748

perineural invasion 1465

peripheral blood leukocyte DNA 2765

personalised medicine $1169,1950,2178$,

2269, 2441

personalised therapy 479

personality 1820

PET 1985

PET-CT 363

petroleum workers 783

P-glycoprotein (P-gp/MDR1) 2669

Pgp 2896

pharmacodynamics 1977

pharmacogenomics 304

pharmacokinetic study 2655

pharmacokinetics $888,894,1977,2170$

pharmacokinetics and

pharmacodynamics 2441

pharyngitis 2796

phase I 2434

phase I/II clinical trial 2389

phosphocholine 330, 625

photodynamic therapy 1698

physical activity 1862

physical function 1409

PI3K 1045, 2081

PI3K expression 1968

PI3K7mTOR inhibitors 2887

PI3K/AKT/MDM2 2479

PI3K/AKT/mTOR 2479

piperlongumine 899

plasma 392, 1001, 2291

plasma miRNAs 976 platelet-to-lymphocyte ratio 2524

platinum 2881

Plk inhibitor 2434

PLK1 510

PLR 1930

pluripotent 2131

polypeptide $N$-acetylgalactosaminyl

transferase-5 (GalNAc-T5) 2021

polyunsaturated fat 1392

population based 868

population pharmacokinetic model 304

population-based cohort 797

potassium intake 797

powerline 1402

PPP2CA 2000

pre-diabetes 2361

predictive 1606

predictive biomarker $320,984,1525$

predictive factors 2195

predictive markers 1169

predictive testing 565

premenopausal 1908

preoperative AFU 1811

preoperative biopsies 1008

prevalence 2804

prevention 1681

primary care 584

primary cell cultures 2269

primary colorectal cancer 441

prior therapy 2821

progesterone receptor 565

prognosis $115,189,208,230,363,369$

$489,565,679,733,775,967,984$,

$1110,1228,1236,1465,1535$,

$1545,1552,1561,1595,1614$,

$1673,1744,1759,1778,1847$

1878, 1943, 2011, 2040, 2081,

$2090,2159,2250,2283,2524$

2531, 2551, 2560, 2583, 2905,

2914, 2923, 2975

prognosis marker 1811

prognostic 1606, 2021

prognostic and predictive factor 2450

prognostic biomarker 1525, 2054

prognostic factors $183,1465,1712,2030$,

$2165,2427,2506$

prognostic index 1688

prognostic marker 399, 435, 991, 2047

prognostic model 42, 2090, 2261

prognostic significance 375

prognostic value 501

prognostics 976

prognostic-score model 297

progression-free survival 1433, 1917, 2700

prokineticin 1446

proliferation 1260, 1446, 2116, 2747, 2847

prophylaxis 1427

prospective cohort 797

prospective cohort study 242

prospective study $530,792,2772$,

prostate cancer 792, 831, 1278, 2000,

2099, 2217, 2389, 2396, 2405, 2593, 2829

prostate cancer mortality 2829

prostate cancer prognosis 1655

prostate cancer stem cells 2593

prostate-specific antigen 2829

proteomic 2277

proteomics 123, 1622, 1748, 2489

PSA screening 2405

psychosocial 593

psychosomatic medicine 1820

PTEN 384, 2874
PTEN loss 1968

pulmonary 1427

pulmonary embolism 1118

pulmonary metastasis 1110

PVT-1 164

qualitative 1681, 1705

quality 1936

quality improvement 850

quality of care 551

quality of life 868

quantitative reverse transcription polymerase chain reaction (qRT-PCR) 991

Rac1 706, 1307, 2677

race 2796

RAD21 1606

radiation 214,1472

radiation therapy 2655

radiation-induced cancer 2354

radical resection 733

radiological features 2633

radiotherapy 256, 501, 908, 1420, 2217

$2389,2623,2633$

randomised controlled study 842

rapid genetic counselling and testing 1081

Ras 1298

reactive oxygen species (ROS) 2569

rectal cancer $19,430,817$

recurrence 19, 1456, 2935

reduction 928

reduction mammaplasty 788

refinement (3Rs) 928

registHER 2756

relapse 2420

relative risk 1663

relative survival 286

renal cancer 616,686

renal cell cancer 797

renal cell carcinoma 1125, 1250, 2250, 2700,2821

renal transplantation 1871

reovirus 83

replacement 928

replication competent HSV-1 94

residential distance at birth 1402

residential history 1855

resistance 479

resistance exercise 831

response biomarker 1727

RET gene rearrangement 1571

retinoblastoma 2623

retreatment 172

retrospective analysis 1125

reverse-phase protein array 2081

reversible posterior leukoencephalopathy syndrome 602

RFS 1811

RhoA 2310

Rho/ROCK 1801

rice 1316

risk $1378,1402,1878$

risk assessment 214

risk classification 2640

risk factor 2738

risk factors and sodium intake 797

risk prediction 535

risk stratification 2405

RNAi 510

ROS 899, 1034

ROS generation 706

ROS, reactive oxygen species 2116

RRM2 1034

RT-PCR 1488

S-1 1163

S100A13 2489

safety 1721

sarcoma 888,1456

sarcoma

screening 1834, 2187, 2412, 2829

second-line 2165

second-line docetaxel 2662

secretome 123

securin 290

sedentary behaviour 817

Se-methylselenocysteine 1733

seminoma 256, 2604, 2738

sentinel node 2837

SERPINB3 2708

serum 2277

sex hormone-binding globulin 1898

sFRP1 1645

short-term survivors 2756

SHP-2 2232

sialophorin 146

side-population 2063

signet ring cell cancer 1712

single-nucleotide polymorphism 1673

sirtuin inhibitor 656

sisters 1074

skin 199

skin cancer 520, 802, 1855

Skp2 1958

slice cultures 479

Smad4 946, 1645

small cell 908

small RNA 2738

small-interfering RNA 2250

smoking 242, 1359, 1385, 2339

SNP 1088

SNTA1 706

socio-economic circumstances 1705

soft tissue sarcoma 2420

solid tumours 2434

soluble CD146 1236

somatic mutations 2812

somatostatin 2232

somatostatin receptor 2115

sorafenib $894,1148,1433,2655,282$

squamous cell carcinoma 199, 1855,

2914

squamous cell lung cancer (SCC) 991

SSTR4 2232

stage I 2187

staging 2544

standardised incidence ratio 788,1871

STAT3 133, 1014, 1767

statin 802

stem cells 1211

stroma 441, 1744

sunitinib 616, 1125, 1433, 2441, 2700

sunlight 1058

surgery 1712

surveillance 1871

survival 115, 208, 489, 556, 908, 1446,

1456, 1545, 1930, 2081, 2531, 2772

survival analysis 1378, 2935

survivin 199

survivors 1338, 1342

susceptibility 1088

symptom recognition 12

symptoms 584

synchronous colorectal cancer 1228 
tamoxifen 1681, 2874

TAp63 2310

targeted therapies 1155, 1169, 2887 targeted molecular therapy 1917

technetium 2837

teenage 1338

temozolomide 2655

temperature 928

temporal lobe injury 49

temporal lobe necrosis 2633

TERT 1058

testis 2738

testosterone 1898

testosterone levels 2201

TGF- $\beta 1,1545,1767$

$\mathrm{T}_{\mathrm{H} 1}$ phenotype 2955

therapeutic drug monitoring 2441

therapeutic target 2677

therapy 1189

therapy resistance 2063

thromboembolism 1118

thymidylate synthase 2728

thyroid 1322

tissue culture 2604

tissue microarray 946, 2040

TMPRSS4 764

TNF 1488

TNM stage system 2109

tongue cancer 2506

tongue squamous cell carcinoma 695

tonsillectomy 2348

toxicity profile 1733

TP53 2874
TRAIL 656

trans fat 1392

transendothelial migration 2099

transforming growth factor-beta

(TGF- $\beta$ ) 156, 724, 946, 2569, 2708

transitional cell carcinoma 26, 1759

translation therapeutics 2450

trastuzumab 1139, 1163, 1472, 2209

trastuzumab-based therapies 1968

treatment 1427

treatment outcome 1721

treatment resistance 2677

Treg 1552

triple negative breast cancer 1045, 1413, 1497,2865

tritium 214

tropomyosin-related kinase B 2923

tumour markers 1250

tumoural 1778

tumour 199, 535, 1269

tumour burden 928

tumour characteristics 1378

tumour graft 1481

tumour heterogeneity 2195

tumour infiltrating leukocyte 1446

tumour location 1595

tumour microenvironment 1199, 2955

tumour recurrence 1001, 2677, 2708

tumour size prediction 1517

tumour suppressor 353, 2144

tumour viscoelasticity 1727

tumour volume 363

tumour-associated macrophages 686 tumour-infiltrating inflammation 1595 tumour-infiltrating lymphocytes 489 ,

$$
501,2560
$$

tumour-initiating capacity 2514

tumour-related circulating miRNA 1001

tumour-stroma ratio 1744

Twist1 958

two-stage design 1950

tyrosine kinase inhibitor 320, 1148, 2655

tyrosine kinases 2479

ubiquitination 1785

UK 4

ultrastaging 2837

ultraviolet 1855

update 535

upper tract urothelial carcinoma 2531

uptake 1681

urgent referral 584

urinary biomarkers 1622

urine 679

urothelial cancer 26

urothelial carcinoma 1759

user interface 535

uterine leiomyoma 2246

UV exposure 1058

vaccine 2804

vaginal cancer 1561

validation study 2531

vascular disrupting agent 2170

vascular endothelial growth factor receptor tyrosine kinase inhibitor 2647 vascular targeting agents 1727

vascularisation 2855

vasculogenesis 1179

vegetable 792

VEGF 469, 1433

VEGF inhibitor 1917

VEGF receptor inhibitor 2821

VEGF-A 2700

venous invasion 19

verteporfin 1698

vincristine 1189

visitatie 850

vitamin D 2772

volasertib 2434

vulval cancer 2837

warning signs 12

WNT5A 1634

women 2321

$\mathrm{X}$-chromosome-linked inhibitor of apoptosis (XIAP) 2881

xCT 2506

XELOX 1438

xenograft 1977, 2855, 2975

young adult 1342

young women 1841

ziv-aflibercept 602

ZNF143 2583

ZNF217 2985 